\title{
Posibilidades de la Armonización de la Educación Superior en Centroamérica: El Caso de la Carrera de Educación Comercial de la UPNFM
}

\author{
Zulema Mercedes Fiallos ${ }^{2}$, Ivy Lou Green y Nolvia Rodriguez \\ Universidad Pedagógica Nacional Francisco Morazán \\ Palabras clave: $<$ Educación Superior $><$ Educación comercial $>$ \\ $<$ Educación Superior Centroamericana $>$ \\ $<$ Armonización de la Educación>
}

\section{Introducción}

En el 2008, El Sistema Centroamericano para la Armonización de la Educación Superior SICEVAES, realizó el primer Seminario-taller Centroamericano sobre Armonización e Integración Académica Regional de la Educación Superior, en el que participaron las universidades públicas pertenecientes al Consejo Superior Centroamericano CSUCA y algunas universidades privadas invitadas de Guatemala, Nicaragua, El Salvador, así como otros invitados de organismos regionales vinculados con este tema.

En octubre del 2010, en la ciudad de Guatemala, se realiza el primer seminario-taller para discutir la propuesta de armonización de la carrera de Administración de Empresas; en el que participaron directores, coordinadores de ésta carrera y miembros de SICEVAES en representación de las universidades públicas vinculadas al CSUCA y universidades privadas invitadas.

\footnotetext{
${ }^{1}$ Este artículo se basa en una ponencia presentada en el 2010 en el IV Congreso del CSUCA realizado en la ciudad de León, Nicaragua.

${ }^{2}$ Participaron en ese momento: Zulema Mercedes Fiallos, Miembro del SICEVAES-CSUCA; Ivy Lou Green Arrechavala, Directora de Evaluación y Acreditación,, Nolvia Rodríguez, Docente de la Carrera de Administración de Empresas.
} 
La Universidad Pedagógica Nacional Francisco Morazán como miembro del SICEVAES participó en el taller mencionado con la carrera de Educación Comercial, por contemplar en su plan de estudios, en el ámbito de la especialidad con un área contable, un área administrativa, un área financiera y un área jurídica; aunque no sea propiamente una Carrera de Administración de Empresas.

Este artículo presenta una propuesta de armonización desde el plan de estudios de la carrera de Educación Comercial, tomando como referencia los ámbitos curriculares consensuados en el taller de armonización mencionado líneas arriba y las acciones definidas para la armonización de la carrera de Administración de empresas.

\section{Antecedentes}

En indudable que la creación del Espacio Europeo de Educación Superior (EEES) marcó las grandes transformaciones en materia de unificación de la educación superior, que son objeto de discusión en diferentes regiones y países del mundo. A continuación se menciona los principales acuerdos que en los 20 años sentaron las bases de este proceso de creación del Espacio Europeo de Educación Superior:

Cuadro No. 1 La evolución de los Acuerdos para la creación del EEES acuerdos indica que no es un proceso propiamente de la Unión Europea, sino que surge de algunos estados miembros, que en un ínterin de dos décadas atrajo la confluencia de 46 países dentro de un esquema de cooperación. No obstante, el proceso está apoyado por la Unión Europea y la

\begin{tabular}{|l|l|}
\hline Año & \multicolumn{1}{|c|}{ Acuerdos / Reuniones } \\
\hline 1988 & Carta Magna de las Universidades Europeas \\
\hline 1997 & Convención de Lisboa \\
\hline 1998 & Declaración de la Sorbona (4 países) \\
\hline 1999 & Declaración de Bolonia (29 países) \\
\hline 2001 & Declaración de Praga (32 países) \\
\hline 2003 & Declaración de Berlín (33 países) \\
\hline 2006 & Declaración de Bergen (45 países) \\
\hline 2007 & Declaración de Londres (46 países) \\
\hline
\end{tabular}
arquitectura institucional de ésta sirve a los Estados para llevarlo a cabo.

Se destaca en este recorrido la Declaración de Bolonia de 1998, que 
Posibilidades de la Armonización de la educación Superior en Centroamérica:

El Caso de la Carrera de Educación Comercial de la UPNFM

firmaron en París, los Ministros de Educación de Francia, Reino Unido, Italia y Alemania, con el propósito de armonizar el diseño del sistema de educación superior europeo, en la que se establecen como objetivos fundamentales los siguientes:

a. Adoptar un sistema de títulos fácilmente comprensible y que pueda ser comparable, promocionando el Suplemento al Diploma.

b. Adoptar un sistema estructural para la enseñanza superior organizado en dos ciclos fundamentales: Grado y Posgrado.

c. Establecer un sistema de créditos compartido (sistema ECTS -European Credit Transfer System- Sistema Europeo de Transferencia de Créditos-).

d. Promover la movilidad, eliminando los obstáculos para el ejercicio efectivo del libre intercambio de estudiantes, profesores, investigadores y personal de administración y servicios, prestando especial atención al acceso a oportunidades de estudios y formación y a los servicios relacionados y al reconocimiento y la valoración de períodos de estudio en el extranjero.

e. Promover la cooperación europea parra asegurar la calidad educativa en la enseñanza superior.

f. Promover la dimensión europea de la enseñanza en la educación superior.

Y todo ello "en un breve plazo de tiempo, y en cualquier caso dentro de la primera década del tercer milenio" (párrafo 10, Declaración de Bolonia,1998). Eso significa que el proceso deberá estar terminado para el año 2010.

La declaración destaca el imprescindible papel de las universidades en la consolidación y desarrollo de las dimensiones intelectuales, culturales y sociales del continente europeo. En conclusión, desde su creación el Espacio Europeo de Educación Superior (EEES) se ha convertido en un referente mundial en los temas de armonización de estudios, pertinencia social, movilidad académica y en general en la mejora de la calidad de la educación superior.

Paralelamente, la idea de un Espacio Latinoamericano se ha discutido también en varios foros. Una iniciativa de esta naturaleza requiere de 
un recorrido similar al desarrollado en Europa, que incluya tanto, acuerdos entre los actores políticos como entre los actores académicos, respecto al modelo educativo, análisis de buenas prácticas, desarrollo de la investigación, estándares comunes y sobre todo la identificación de espacios comunes para lograr el correspondiente reconocimiento en la sociedad.

La experiencia de construcción del Espacio Europeo de Educación Superior (EEES) y su traducción euro-latinoamericana, Tuning América Latina, en el 2004, significó un fuerte impulso a estas iniciativas, propició los espacios de reflexión para identificar los elementos de referencia que sustentaran los puntos de acuerdo para encontrar la convergencia dentro de las estructuras educativas. "Estos elementos son claves para tender los puentes para el reconocimiento de las titulaciones" (Gonzales y otros, 2007:11). Al 2007 se dejan identificadas las bases para la definición del perfil de egreso basada en competencias y una metodología que permita la articulación de los sistemas de educación superior de América Latina.

Se destacan el esfuerzo de la Organización Universitaria Interamericana (OUI) a través de su programa CAMPUS, quien ha sentado las bases para construir un Espacio Latinoamericano y del Caribe de Educación Superior, denominado: ENLACES, definido como instrumento de integración académica y de investigación entre las universidades miembros y otras del continente. La propuesta busca el establecimiento de un sistema de créditos y criterios comunes de armonización curricular que permiten fijar estándares, generar confianza interinstitucional y convergencia, y promover la movilidad entre las instituciones de educación superior de la región latinoamericana.

ENLACES es la conjunción de esfuerzos de la OUI y la Unión de Universidades de América Latina y el Caribe (UDUAL), quienes a través de su Junta Directiva y Consejo Ejecutivo, proponen los siguientes objetivos: ${ }^{3}$

${ }^{3} \odot 2011$ ENLACES Espacio Latinoamericano y del Caribe de Educación Superior. 


\section{Objetivo General}

La Construcción de (ENLACES), está basado en acuerdos políticos y académicos, estrategias operativas y el fomento masivo de la ciencia y sus actores.

\section{Objetivos específicos}

1. Diseño, creación y puesta en marcha del Espacio Latinoamericano y del Caribe de Educación Superior ENLACES.

2. Desarrollo y aplicación masiva de un modelo de Incubadoras de Investigación y doctorados colaborativos en las IES latinoamericanas para el fomento de la ciencia.

La UDUAL y la OUI han conformado una Comisión Técnica que es la encargada de llevar la agenda política y académica: ${ }^{4}$

- Diseño, publicación, distribución de documentos metodológicos de soporte de ENLACES;

- Un sistema de grados académicos fácilmente comprensibles y comparables;

- Un sistema de acumulación y transferencia de créditos;

- La movilidad de los estudiantes, docentes e investigadores;

- La cooperación en lo que respecta a la garantía de la calidad;

- La dimensión Latinoamericana y del Caribe de la enseñanza superior:

- La formación continua;

- La participación de los centros de enseñanza superior y los estudiantes para crear de manera constructiva el ENLACES.

- Necesidad de la investigación, la formación en investigación y el fomento de la interdisciplinariedad para mantener y mejorar la calidad de la enseñanza superior y reforzar su competitividad.

- La puesta en práctica de las referencias y las directrices para la garantía de la calidad;

- Los marcos nacionales de cualificaciones;

- La expedición y el reconocimiento de los títulos conjuntos, incluidos los de doctorado; 
- La creación de oportunidades para itinerarios flexibles de formación en la enseñanza superior, incluyendo la existencia de disposiciones para la validación de los conocimientos adquiridos.

Todos estos esfuerzos muestran el interés de universidades y académicos, convirtiéndose en decisivos acercamientos en la búsqueda de la armonización de la educación superior en la región.

\section{El Espacio de Armonización de los Sistemas de Educación Superior Centroamericano.}

El CSUCA ha impulsado desde su creación la calidad de la educación superior a través de la estrategia de la evaluación y acreditación institucional y de programas. Se han realizado distintas acciones; desde convenios, declaraciones, proyectos, iniciativas, cursos, talleres, creación de agencias, foros, con el objetivo de alcanzar y poner en práctica procesos de armonización académica.

Ya en 1962, en el Plan de Integración Regional de la Educación Centroamericana, PIRESC I, en sus cinco objetivos se evidencia el interés por la armonización en la educación superior pública de la región; reconocimiento de estudios, grados y títulos, definición de competencias con base en la propuesta Tunnig para América Latina, homologación de la unidad académica y el programa de intercambio $\mathrm{d}$ académica de profesionales y estudiantes, entre otros.

Con el Programa de Apoyo a la Integración Regional Centroamericana (PAIRCA), el CSUCA realizó un estudio cuyo resultado fue una propuesta de armonización de los sistemas de educación superior de los países centroamericanos. Proponiéndose como objetivo: “Delinear los pasos a seguir para la armonización, integración y coordinación regional de los sistemas de educación superior en un espacio común centroamericano" ${ }^{5}$

Las recomendaciones que resultaron de ese estudio, destacan que para el funcionamiento del proceso de armonización regional se requiere:

${ }^{5}$ Batista (2010), Análisis comparativo y propositivo para la Armonización de los Sistemas de Educación Superior. 
a. La integración de una comisión de armonización que dé seguimiento y estimule el proceso en cada universidad miembro, bajo la responsabilidad del SICEVAES.

b. Generar condiciones para el procesamiento y sistematización de información. Red Centroamericana de Información (RIESCA).

c. El seguimiento y monitoreo a cargo del Comité de Coordinación Regional del SICEVAES-CCR.

En el cuadro siguiente se presenta un resumen de la ruta a seguir en este proceso de armonización, estructurada en tres bloques: los principios en que se basa este proceso, los objetivos estratégicos y los medios, instrumentos y acciones que se han propuesto.

Cuadro 2

Principios, objetivos e instrumentos del espacio Centroamericano de la Educación Superior

\begin{tabular}{|c|l|l|}
\hline \multicolumn{1}{|c|}{ Principios } & \multicolumn{1}{|c|}{$\begin{array}{c}\text { Objetivos } \\
\text { Estratégicos }\end{array}$} & \multicolumn{1}{c|}{ Medios e Instrumentos y acciones } \\
\hline $\begin{array}{l}\text { Calidad de la } \\
\text { educación su- } \\
\text { perior }\end{array}$ & $\begin{array}{l}\text { Garantía de Calidad del } \\
\text { Sistema de educación su- } \\
\text { perior }\end{array}$ & $\begin{array}{l}\text { Suscripción de un convenio regional entre } \\
\text { universidades miembros del CSUCA } \\
\text { Autoevaluación y Evaluación Externa } \\
\text { institucional y de programas } \\
\text { Monitoreo a los procesos de mejora } \\
\text { institucional y de programas académicos } \\
\text { Fortalecimiento de las agencias de } \\
\text { acreditación } \\
\text { Diseño y divulgación de documentos } \\
\text { metodológicos de soporte }\end{array}$ \\
\hline $\begin{array}{l}\text { Respeto a la } \\
\text { autonomía }\end{array}$ & Armonización ${ }^{6}$ & $\begin{array}{l}\text { Comprensión y convergencias de las } \\
\text { estructuras educativas } \\
\text { Adopción de un sistema de libre adhesión, } \\
\text { periódico, de aplicación gradual y experi- } \\
\text { mental. } \\
\text { Creación de oportunidades para itinerarios } \\
\text { flexibles de formación en la enseñanza su- } \\
\text { perior } \\
\text { Homologar en su contenido curricular los } \\
\text { planes de estudio }\end{array}$ \\
\hline
\end{tabular}

6 “Construcción de un espacio común de la educación superior, que permita además de la búsqueda de consensos , facilitar el reconocimiento de las calificaciones y competencias de las personas, tanto para continuar con sus estudios y su vida laboral como para incrementar la movilidad en la región centroamericana." , CSUCA/ PAIRCA. 


\begin{tabular}{|c|c|c|}
\hline \multirow[t]{3}{*}{ Consenso } & Homologar & $\begin{array}{l}\text { Poner en sintonía las estructuras educativas: } \\
\text { niveles y grados académicos. } \\
\text { Acordar requisitos de ingreso y perfil de } \\
\text { egreso en las universidades } \\
\text { Crear el sistema de crédito } \\
\text { Centroamericano: } \\
\text { Armonizar a } 45 \text { horas el crédito académico. } \\
\text { Homogeneizar a } 15 \text { semanas el ciclo } \\
\text { académico } \\
\text { El rango de la hora-clase, } 50-60 \text { minutos. } \\
\text { Valorar el trabajo independiente del } \\
\text { estudiante en una relación de dos horas por } \\
\text { hora-clase. }\end{array}$ \\
\hline & Reconocer títulos & $\begin{array}{l}\text { Equiparación de programas basados en el } \\
\text { reconocimiento de componentes } \\
\text { curriculares, aprobados en un 50\%. } \\
\text { Aprobar y reconocer hasta un } 70 \% \text { los } \\
\text { cursos de los cursos de una carrera } \\
\text { determinada. } \\
\text { Reconocimiento de los cursos realizados } \\
\text { en procesos de movilidad. }\end{array}$ \\
\hline & $\begin{array}{l}\text { Elaborar el documento } \\
\text { complementario al diploma }\end{array}$ & $\begin{array}{l}\text { Construir un formulario que avale la } \\
\text { formación recibida y su ámbito laboral }\end{array}$ \\
\hline
\end{tabular}

Fuente: Elaboración propia en base al documento CSUCA / PAIRCA

A la fecha (2013) ya existe consenso en el documento complementario al título y ha sido aprobado por las autoridades de las diferentes universidades.

\section{III.Propuesta de armonización de la carrera de educación comercial}

Se inicia este apartado con un cuadro comparativo entre las competencias definidas en el proyecto TUNNIG-Latinoamérica para la carrera de Administración de Empresas y las competencias definidas en el plan de estudios de la carrera de la Licenciatura en Educación comercial de la UPNFM.

A continuación se presenta una propuesta de los espacios pedagógicos en los que se puede ofrecer intercambio y movilidad docente y estudiantil, con base en las fortalezas del plan de estudios de

\footnotetext{
7 "Un crédito académico o unidad valorativa se define como la unidad de medida de la carga académica del aprendizaje del estudiantes que es igual a 45 horas aplicadas a una actividad supervisada, evaluada y aprobada por el docente.", CSUCA/PAIRCA
}

<PARADIgMA> - Revista de Investigación Educativa. Año 20. No. 31 
Posibilidades de la Armonización de la educación Superior en Centroamérica:

El Caso de la Carrera de Educación Comercial de la UPNFM

infraestructura de la UPNFM; y de las oportunidades para la carrera de administración de empresas de las universidades centroamericanas.

Cuadro 3

Comparativo entre competencias TUNNING-UPNFM según áreas de especialidades

\begin{tabular}{|c|c|c|c|}
\hline \multirow[b]{2}{*}{ AREA } & \multirow[b]{2}{*}{ TUNNING } & \multicolumn{2}{|c|}{ UPNFM } \\
\hline & & Competencias & $\begin{array}{c}\text { Espacios } \\
\text { Pedagógicos }\end{array}$ \\
\hline $\begin{array}{l}\text { Área Contable } \\
\text { El área contable es la } \\
\text { preparación fundamental } \\
\text { de la especialidad, } \\
\text { comprende los principios } \\
\text { y normas de contabilidad } \\
\text { vigentes, la apertura y } \\
\text { cierre del ciclo contable } \\
\text { de empresas relacionadas } \\
\text { con la compra y venta de } \\
\text { productos, prestación de } \\
\text { servicios, transformación } \\
\text { de bienes y venta de } \\
\text { productos intangibles. }\end{array}$ & 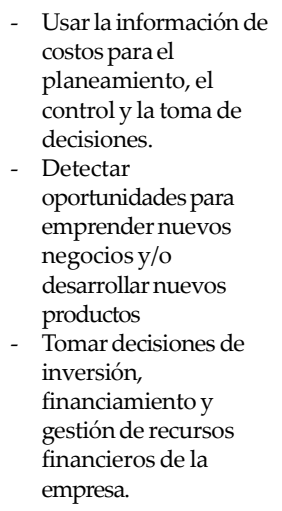 & $\begin{array}{l}\text { - Capacidad para realizar } \\
\text { el proceso contable, } \\
\text { de acuerdo a las formas } \\
\text { y procedimientos } \\
\text { establecidos por la } \\
\text { legislación nacional y } \\
\text { lasnormas } \\
\text { internacionales de } \\
\text { contabilidad, } \\
\text { información financiera } \\
\text { y auditoría. }\end{array}$ & $\begin{array}{l}\text { Contabilidad I } \\
\text { Contabilidad II } \\
\text { Contabilidad III } \\
\text { Contabilidad de Costos I } \\
\text { Contabilidad de Costos II } \\
\text { Operaciones Bancarias } \\
\text { Seminario Contable. }\end{array}$ \\
\hline $\begin{array}{l}\text { Área Administrativa } \\
\text { En el área administrativa } \\
\text { se ubican los espacios de } \\
\text { formación que están } \\
\text { vinculados con la gestión } \\
\text { empresarial, } \\
\text { incluyéndose la } \\
\text { economía, administración } \\
\text { y las ramas auxiliares de la } \\
\text { misma, como ser; las } \\
\text { relaciones } \\
\text { interpersonales, la } \\
\text { gestión de la calidad, la } \\
\text { informática y su } \\
\text { vinculación al quehacer } \\
\text { profesional. }\end{array}$ & $\begin{array}{l}\text { - Administrary } \\
\text { desarrollar el talento } \\
\text { humano en la } \\
\text { organización } \\
\text { - Desarrollar, } \\
\text { implementar y } \\
\text { gestionar sistemas de } \\
\text { control administrativo. } \\
\text { - Identificar las } \\
\text { interrelaciones } \\
\text { funcionales de la } \\
\text { organización } \\
\text { - Utilizar las } \\
\text { tecnologías de } \\
\text { información y } \\
\text { comunicación en la } \\
\text { gestión } \\
\text { - Desarrollar un } \\
\text { planeamiento } \\
\text { estratégico, táctico y } \\
\text { operativo integral con } \\
\text { baseen análisis micro } \\
\text { y macroeconómico. } \\
\text { - Tomar decisiones de } \\
\text { inversión, } \\
\text { financiamiento y } \\
\text { gestión de recursos } \\
\text { financieros de la }\end{array}$ & $\begin{array}{l}\text { - Capacidad para uso, } \\
\text { análisise } \\
\text { interpretación de } \\
\text { documentos de } \\
\text { carácteradministrativo } \\
\text { y financiero para la } \\
\text { toma de decisiones en } \\
\text { las distintas } \\
\text { modalidades } \\
\text { empresariales. } \\
\text { - Capacidad para aplicar } \\
\text { la tecnología al } \\
\text { procesoadministrativo } \\
\text { - contable. } \\
\text { Capacidad para } \\
\text { identificar los } \\
\text { elementos del } \\
\text { entorno económico, } \\
\text { legal, social, político, } \\
\text { gubernamentaly } \\
\text { cultural de las } \\
\text { empresas y del país, } \\
\text { logrando con ello el } \\
\text { desarrollo de un } \\
\text { pensamiento crítico } \\
\text { ante la realidad } \\
\text { nacional e } \\
\text { internacional. }\end{array}$ & $\begin{array}{l}\text { Administración General } \\
\text { Gestión del talento } \\
\text { humano } \\
\text { Mercadotecnia } \\
\text { Estadística Aplicada a la } \\
\text { Administración } \\
\text { Gestión de la Calidad } \\
\text { Seminario Tributario } \\
\text { Auditoría } \\
\text { Formulación y } \\
\text { Evaluación de Proyectos } \\
\\
\text { Sistemas Administrativos } \\
\text { y contables. } \\
\text { Economía. } \\
\text { Informática General } \\
\text { Informática Aplicada I }\end{array}$ \\
\hline
\end{tabular}




\begin{tabular}{|c|c|c|c|}
\hline & 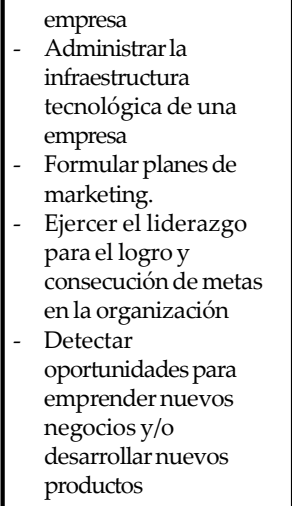 & $\begin{array}{l}\text { - Capacidad para atender } \\
\text { las distintas áreas de la } \\
\text { administración en lo } \\
\text { relacionado a la } \\
\text { investigación, } \\
\text { formulación y } \\
\text { evaluación de } \\
\text { proyectos } \\
\text { empresariales. }\end{array}$ & \\
\hline $\begin{array}{l}\text { Área Financiera } \\
\text { Se presenta el } \\
\text { componente de las } \\
\text { herramientas financieras } \\
\text { relacionadas con la toma } \\
\text { de decisiones } \\
\text { empresariales, incluye los } \\
\text { elementos de cálculos } \\
\text { matemáticos, el análisis } \\
\text { financiero y el entorno } \\
\text { corporativo de las } \\
\text { empresas. El área } \\
\text { financiera es auxiliar de } \\
\text { los conocimientos del } \\
\text { área contable. }\end{array}$ & $\begin{array}{l}\text { - Tomar decisiones de } \\
\text { i n e r s i ó n , } \\
\text { financiamiento y } \\
\text { gestión de recursos } \\
\text { financieros de la } \\
\text { empresa. } \\
\text { - }\end{array}$ & \begin{tabular}{|l|} 
- Capacidad para \\
resolver los problemas \\
matemáticos \\
vinculados con la \\
contabilidad, finanzas \\
y administración para \\
la toma de decisiones \\
empresariales. \\
- Capacidad para uso, \\
análisise \\
interpretación de \\
documentos de \\
carácter administrativo \\
y financiero para la \\
toma de decisiones en \\
las distintas \\
modalidades \\
empresariales.
\end{tabular} & $\begin{array}{ll}\text { - } & \text { Matemáticas } \\
& \text { Mercantil } \\
\text { - } & \text { Matemática } \\
& \text { Financiera } \\
\text { - } & \text { Administración } \\
& \text { Financiera }\end{array}$ \\
\hline $\begin{array}{l}\text { Área Jurídica } \\
\text { Contiene los aspectos } \\
\text { relacionados con el } \\
\text { quehacer empresarial, } \\
\text { incluye los principios } \\
\text { fundamentales de } \\
\text { derecho, las leyes } \\
\text { tributarias vigentes y } \\
\text { aplicables en el ámbito } \\
\text { empresarial y las leyes } \\
\text { educativas vinculadas con } \\
\text { el quehacer docente. }\end{array}$ & $\begin{array}{l}\text { - Evaluar el marco } \\
\text { jurídico aplicado a la } \\
\text { gestión empresarial. }\end{array}$ & $\begin{array}{l}\text { - Capacidad para } \\
\text { realizar el proceso } \\
\text { contable, de acuerdo a } \\
\text { las formasy } \\
\text { procedimientos } \\
\text { establecidos por la } \\
\text { legislación nacional y } \\
\text { lasNormas } \\
\text { Internacionales de } \\
\text { contabilidad, } \\
\text { información financiera } \\
\text { y Auditoría. }\end{array}$ & $\begin{array}{l}\text { Legislación I } \\
\text { Legislación II }\end{array}$ \\
\hline $\begin{array}{l}\text { Práctica Profesional de } \\
\text { Educación Comercial } \\
\text { Se ubica en una etapa } \\
\text { previa a la etapa terminal } \\
\text { de la formación de los } \\
\text { educandos, con ello se } \\
\text { pretende que las } \\
\text { competencias adquiridas } \\
\text { durante el proceso de } \\
\text { enseñanza-aprendizaje }\end{array}$ & $\begin{array}{l}\text { - Elaborar, evaluary } \\
\text { administrar proyectos } \\
\text { empresariales en } \\
\text { diferentes tipos de } \\
\text { organización }\end{array}$ & $\begin{array}{l}\text { - Capacidad para realizar } \\
\text { el proceso contable, de } \\
\text { acuerdoa las formas y } \\
\text { procedimientos } \\
\text { establecidos por la } \\
\text { legislación nacional y } \\
\text { las Normas } \\
\text { Internacionales de } \\
\text { contabilidad, } \\
\text { información financiera } \\
\text { y Auditoría. }\end{array}$ & $\begin{array}{l}\text { - Práctica Administrativa } \\
\text { Contable. }\end{array}$ \\
\hline
\end{tabular}


Posibilidades de la Armonización de la educación Superior en Centroamérica: El Caso de la Carrera de Educación Comercial de la UPNFM

\begin{tabular}{|c|c|}
\hline $\begin{array}{l}\text { sean afianzadas con una } \\
\text { práctica real, misma que } \\
\text { será desempeñada en las } \\
\text { oficinas de contabilidad, } \\
\text { administración y finanzas } \\
\text { y áreas relacionadas con } \\
\text { la educación comercial. }\end{array}$ & $\begin{array}{l}\text { - Capacidad para } \\
\text { resolver los } \\
\text { problemas } \\
\text { matemáticos } \\
\text { vinculados con la } \\
\text { contabilidad, la } \\
\text { administración, la } \\
\text { auditoría y las } \\
\text { finanzas. } \\
\text { - Capacidad para uso, } \\
\text { análisise } \\
\text { interpretación de } \\
\text { documentos de } \\
\text { carácter } \\
\text { administrativoy } \\
\text { financiero para la } \\
\text { toma de decisiones } \\
\text { en las distintas } \\
\text { modalidades } \\
\text { empresariales. } \\
\text { - Capacidad para } \\
\text { atender las distintas } \\
\text { áreas de la } \\
\text { administración en lo } \\
\text { relacionado a la } \\
\text { investigación, } \\
\text { formulacióny } \\
\text { evaluación de } \\
\text { proyectos } \\
\text { empresariales. }\end{array}$ \\
\hline
\end{tabular}

Fuente: Elaboración propia en base al documento CSUCA / PAIRCA

El cuadro comparativo muestra que la carrera de Educación Comercial, es comparable sobre la base del acuerdo de considerar las competencias Tuning América Latina como ejes fundantes del diseño curricular de la carrera. Por lo tanto homologable. Sobre esa conclusión se expone a continuación algunos lineamientos para realizar la movilidad académico profesional y de estudiantes, desde la base de las fortalezas de la UPNFM como institución educativa formadora de docentes y de la carrera de educación comercial: especialización en pedagogía y didáctica en la enseñanza de asignaturas del área contable, administrativa, financiera y legislación; dos prácticas profesionales una didáctico-pedagógica y una laboral-empresarial; capacidad física instalada: laboratorios, talleres, recursos bibliográficos tanto en formato impreso como digital y software especializado; infraestructura tecnológica y de comunicación; y la experiencia en el desarrollo curricular basado en competencias. 


\section{Comentarios Finales}

En nuestro "Espacio Centroamericano" aún se requiere de espacios de reflexión para la búsqueda de consensos, que nos lleven por el camino recorrido en la construcción de la "europa del conocimiento" (EEES); que no surge aislada sino dentro de un marco social, económico y político en el cual prima la idea del "fermento de una comunidad mas amplia y mas profunda" (Shuman, 1950) y como se lee en el Tratado de la Unión Europea (Consejo Europeo, 1992) "La Unión tiene por finalidad promover la paz, sus valores y el bienestar de sus pueblos", introduce el concepto de ciudadanía europea como un factor irremplazable para el crecimiento social y humano y componente indispensable para consolidar y enriquecer a la ciudadanía europea, "capaz de dar a sus ciudadanos las competencias necesarias para afrontar los retos del nuevo milenio, junto con una conciencia de valores compartidos y pertenencia a un espacio social y cultural común." (Declaración de Bolonia, 1999,3)

En consonancia con todo este movimiento de armonización de la educación superior centroamericano el Consejo Superior Centroamericano CSUCA ha liderado una serie de programas, encuentros, talleres y seminarios que se traducen en una ruta estratégica en los que se han definido objetivos y acciones que ponen de manifiesto el entendimiento en cuanto a:

- El desarrollo de relaciones de mutua confianza entre las instituciones de Educación Superior,

- Acuerdo en una estructura curricular marco sin renunciar a las características propias de cada sistema en particular, hacer converger los fines educativos.

- Búsqueda de los términos de una estructura marco para las cualificaciones, sentar las bases para un reconocimiento de períodos de estudio y de títulos.

- Identificación de principios elementales relativos al sentido y el funcionamiento de dichas instituciones,

- Acuerdo en una estructura curricular marco sin renunciar a las características propias de cada sistema en particular, hacer converger los fines educativos.

- Sentar las bases para un reconocimiento de períodos de estudio y de títulos en términos de una estructura marco para las 
Posibilidades de la Armonización de la educación Superior en Centroamérica:

EI Caso de la Carrera de Educación Comercial de la UPNFM

\section{cualificaciones}

- Acuerdos en una estructura curricular marco sin renunciar a las características propias de cada sistema en particular, hacer converger los fines educativos.

- Identificar carreras piloto para la puesta en marcha de experiencias de armonización: Administración de Empresas, Matemática e Ingeniería Civil. 


\section{Referencias}

Batista (2010), Análisis comparativo y propositivo para la Armonización de los Sistemas de Educación Superior. Recuperado el 10 de enero 2011, disponible en www.csuca.org/ Estudiodearmonizacion/.

Caride Gómez, J. A. (2008). El grado en educación social en la construcción del espacio europeo de educación superior, Universidad de Santiago de Compostela.

EEES. Declaración de Bolonia. Recuperado el 10 de enero 2011. Disponible en www.eees.es/es/eees

ENLACES (2011). Espacio de Encuentro Latinoamericano y Caribeño de Educación Superior. Recuperado el 10 de enero 2011. Disponible en www.oui-iohe.org/campus/eles/en/enlaces/

Fernández Lamarra, N. (2004) Hacia la convergencia de los sistemas de educación superior en América Latina. Recuperado el 10 de enero 2011, disponible en redalyc.uaemex.mx/pdf/800/80003504.

Fuentes Soria J. y otros (2008) Propuesta Armonización de los sistemas de educación superior de los países centroamericanos. CSUCA / PAIRCA

Gonzales, J, Beneitone, P., Esquetini, C., Marty, M., Siufi, G.,Wagenaar, R, (2007). Reflexiones y perspectivas de la Educación Superior en América Latina. Informe final- Proyecto Tuning 2004-2007. Universidad de Deusto, Universidad de Groningen. Bilbao.

Valle, J. (2009). La armonización de la educación superior europea. El espacio europeo de la educación superior.. Revista Avances en Supervisión Educativa. No.10 Disponible en http://adide.org/ revista/index.php?option=com_content\&task=view $\&$ id $=307 \&$ Itemid $=63$ 